\title{
VI. Die Reaktion des Offizierskorps auf die politische Durchdringung der NVA 1956-1961
}

Den größten Widerspruch gegen die politische Durchdringung der NVA und die Priorität des Politischen in der militärischen Sphäre gab es auf Seiten des Offizierskorps. Die Mitgliedschaft in der SED war dabei anscheinend kein Hinderungsgrund. Der Leiter der PKK im Ministerium für Nationale Verteidigung, Oberst Engels, beklagte im September 1956, er habe den Eindruck, daß sich viele Kommandeure nicht als Parteimitglieder fühlten und in ihren Maßnahmen häufig keine „Parteiverbundenheit" zum Ausdruck brächten¹. Vor allem die militärische Truppenführung war von der Gründung der NVA an bemüht, ihre fachliche Autonomie zu sichern und die vielfältigen Interventionen der SED in die militärischen Diskussions- und Entscheidungsprozesse so weit wie möglich zu unterbinden.

Entsprechend ihrer herausgehobenen Stellung im System der politischen Durchdringung der Streitkräfte richtete sich die Kritik aus dem Offizierskorps in den ersten zwei Jahren des Bestehens der NVA besonders gegen die Politorgane. Die ZKAbteilung für Sicherheitsfragen äußerte 1957: „Es ist bekannt, dass es zur Zeit in unseren Einheiten nicht wenig Diskussionen gibt, welche die Zweckmäßigkeit der Politorgane in unserer Armee anzweifeln und verschiedentlich ihre Liquidierung vorschlagen.“2 Der Politapparat wurde allgemein als „zu groß“ und der „Einfluß der Partei ... auf die militärische Ausbildung" als "nicht notwendig" erachtet ${ }^{3}$. Bei der Mehrzahl der Offiziere bestand zudem wenig Neigung, sich die Theorie des Marxismus-Leninismus anzueignen. Statt dessen versuchten sie häufig, sich der politischen Weiterbildung und dem gesellschaftswissenschaftlichen Unterricht $\mathrm{zu}$ entziehen ${ }^{4}$.

Feststellen läßt sich zudem eine ablehnende Haltung des Offizierskorps gegen die starke Präsenz der Justiz- und Sicherheitsorgane in der NVA. So war die Bereitschaft vieler Kommandeure offenbar gering, mit dem jeweils zuständigen Militärstaatsanwalt zusammenzuarbeiten. Im März 1956 stellte die SED fest: „Leider haben noch nicht alle Kommandeure diese helfende Aufgabe des Staatsanwaltes erkannt und sehen in ihm noch den Quälgeist, der ihnen gerade noch gefehlt hat, wenn nicht Schlimmeres." 5

Auch das Verhältnis der militärischen Truppenführung zu den operativen Mitarbeitern des MfS war infolge deren weitreichender Befugnisse stark angespannt.

1 Protokoll Nr. 7/56 über die Sitzung des Kollegiums des Ministeriums für Nationale Verteidigung, 21. September 1956, BA-MA, VA-01/2030, Bl. 112.

2 Probleme für einen Diskussionsbeitrag zur Beratung von Mitgliedern des Politbüros mit leitenden Parteifunktionären in der NVA, 1957, SAPMO-BArch, DY 30/IV 2/12/9, Bl. 10.

3 Protokoll der Eggersdorfer Tagung, 12./13. Juni 1957, BA-MA, VA-P-01/037, Bl. 29.

4 Protokoll der Delegiertenkonferenz der Parteiorganisationen der SED in der NVA, 24.-26. Juni 1958, BA-MA, VA-P-01/038, Bl. 33.

5 Protokoll der Delegiertenkonferenz der Parteiorganisationen der SED im Dienstbereich des Ministeriums für Nationale Verteidigung, 5.-11. März 1956, BA-MA, VA-P-01/036, Bl. 281. 
Viele verantwortliche NVA-Offiziere versuchten die Offiziere des MfS auf Distanz zu den militärischen Entscheidungsprozessen zu halten. So kritisierte etwa die ZKAbteilung für Sicherheitsfragen nach einem Brigadeeinsatz im Juni 1959: „Die verantwortlichen Offiziere der Nationalen Volksarmee informieren die Mitarbeiter des Ministeriums für Staatssicherheit nicht bzw. wenig über Dinge, die für die Abwehrarbeit ... wertvoll sind."6 Konkret hieß es in bezug auf das 23. Motorisierte Schützenregiment (MSR), der Offizier der Verwaltung 2000 werde von der Regimentsleitung „nicht beachtet“. Man informiere ihn weder über Leitungssitzungen noch über andere Veranstaltungen?.

Im Zuge der Einführung kollektiver Entscheidungsmechanismen nach dem Inkrafttreten der „Instruktion für die Arbeit der Parteiorganisationen der SED in der NVA“ im Mai 1957, spätestens aber nach dem Politbürobeschluß „Über die Rolle der Partei in der NVA" vom 14. Januar 1958, durch den endgültig das kollektive Führungsprinzip in der Armee implementiert wurde ${ }^{8}$, verlagerte sich die Stoßrichtung der Kritik des Offizierskorps an der politischen Kontrolle. Sie zielte jetzt vor allem auf den Einfluß der Parteiorganisationen und -leitungen in rein militärischen Fragen der Truppenführung. So mußte der damalige Leiter der ZK-Abteilung für Sicherheitsfragen Borning im Juni 1958 in der Zeitung „Die Volksarmee“ öffentlich zugeben, daß es nach Bekanntwerden des Politbürobeschlusses „bei leitenden Offizieren Bedenken und Zweifel" gegeben habe, ob die erweiterten Kompetenzen der Parteiorganisationen in militärischen Fragen "nicht etwa zu weit gingen und auf eine Minderung der von der Partei geforderten Schlagkraft und Einsatzbereitschaft der Nationalen Volksarmee hinauslaufen könnten "9. Intern wurde die überwiegend ablehnende Haltung offenbar sogar sehr viel drastischer artikuliert. So äußerte ein Oberleutnant: „... jetzt macht die Partei alles, der Kommandeur ist nur noch Aushängeschild." ${ }^{10}$ Ein anderer Oberleutnant befand: "Jetzt hat jeder Zivilklub (gemeint ist die Partei) die Möglichkeit, in die Arbeit der Armee reinzureden und zu kontrollieren." 11 Viele Kommandeure und leitende Offiziere betrachteten die kollektive Beratung militärischer Entscheidungen demzufolge als massive Beschränkung ihrer eigenen Verantwortung 12.

Ein Teil von ihnen versuchte von vornherein, die zunehmende Einflußnahme der Parteiorganisationen auf die militärischen Diskussions- und Entscheidungsprozesse $\mathrm{zu}$ unterbinden. Im Politbürobeschluß wurde daher auch kritisiert, manche Kommandeure und leitenden Offiziere sähen die Parteiorganisationen "noch immer als Organe an, die ausschließlich zur Unterstützung ihrer Arbeit zur Verfügung" stün-

${ }^{6}$ Erfahrungen und Schlußfolgerungen aus den Brigadeeinsätzen des ZK der SED in der 1. MSD, 4. MSD, in Sonderobjekten und Objekten der Luftverteidigung, 23. Juni 1959, SAPMO-BArch, DY 30/IV 2/12/21, Bl. 244.

7 Ebenda, Bl. 243.

8 Vgl. S. $99 \mathrm{ff}$.

9 Borning, Unter der Führung der Partei erringen wir Erfolge, S. 2.

10 Bericht über die Stimmungen der Armeeangehörigen und Erscheinungen in der politischen Arbeit in der ersten Monatshälfte März 1958, 1958, BA-MA, VA-01/1752, Bl. 75.

11 Bericht über die Stimmungen der Armeeangehörigen und Erscheinungen in der politischen Arbeit in der ersten Monatshälfte April 1958, 1958, BA-MA, VA-01/1752, Bl. 66.

12 Protokoll der 2. Tagung der II. Delegiertenkonferenz der Parteiorganisationen der SED in der NVA, 25./26. März 1959, BA-MA, VA-P-01/556, Bl. 75. 
den und glaubten, „deren Tätigkeit befehlen zu können"13. Besonders die Kommandeure waren bestrebt, die Parteileitungen bei ihrer Führungsarbeit nach Möglichkeit zu umgehen ${ }^{14}$. Andererseits scheinen einige verantwortliche Offiziere aber auch unberechtigt an Sitzungen der Parteileitungen teilgenommen zu haben, die sie vereinzelt sogar störten ${ }^{15}$. Darüber hinaus kam es vor, daß Kommandeure ihre Autorität in der jeweiligen Einheit durch die „Versetzung eines gewählten Parteisekretärs ohne Wissen und Einverständnis der betreffenden Parteiorganisation “ $\mathrm{zu}$ wahren oder wiederherzustellen versuchten ${ }^{16}$.

Vor allem wehrten sich die Offiziere gegen die Anwendung des Prinzips der Kritik und Selbstkritik im Rahmen von Parteiversammlungen in bezug auf ihre Person. Als dieses Erziehungsinstrument seit dem Mai 1957 zur kritischen Bewertung ihrer dienstlichen Tätigkeit herangezogen werden konnte, äußerten offenbar viele Offiziere die nach Auffassung der SED „falsche Einstellung, daß das Prinzip der Einzelleitung, die Befehlsgebung und die Struktur der Armee nicht mit den allgemeinen Prinzipien der Parteiarbeit und der innerparteilichen Demokratie zu vereinbaren “ $\operatorname{seien}^{17}$. Für die leitenden Offiziere war es inakzeptabel, sich vor dem Personalbestand ihrer Einheit für ihre militärische Führungsarbeit öffentlich verantworten und rechtfertigen $\mathrm{zu}$ müssen. Einige waren daher bestrebt, jede Kritik im Ansatz zu unterbinden ${ }^{18}$, indem sie „entweder gar nicht auf die an ihnen geübte Kritik“ reagierten oder versuchten, diese „abzuschwächen“. Teilweise, so die ZK-Abteilung für Sicherheitsfragen, hätten sie die Kritik auch einfach „mit einer Gegenkritik beantwortet" 19 .

Im Zuge der Umwandlung der KVP in eine reguläre Armee entwickelte das Offizierskorps der NVA ein zunehmend professionelles Selbstverständnis. Viele der Offiziere sahen es jetzt offenbar als ihre vorrangige Aufgabe an, die militärische Einsatzfähigkeit der Streitkräfte zu steigern. Zum Mißfallen der SED traten politi-

13 Über die Rolle der Partei in der NVA. Protokoll Nr. 4/58 der Sitzung des Politbüros des ZK der SED, 14. Januar 1958, SAPMO-BArch, DY 30/J IV 2/2/576, Bl. $191 \mathrm{f}$. So wurde etwa auf einer Parteiaktivtagung der Artillerieschule in Dresden mehrheitlich die Forderung erhoben, einige der Absolventen sollten nicht zum Offizier befördert werden, weil ihnen hierfür die politischen und fachlichen Voraussetzungen fehlten. Einzig der Chef der Verwaltung Artillerie des Ministeriums für Nationale Verteidigung, Oberstleutnant Hotzky, widersprach dem, konnte sich mit seiner Auffassung jedoch nicht durchsetzen. Daraufhin ließ er am nächsten Tag die verantwortlichen Parteifunktionäre zu sich kommen und äußerte ihnen gegenüber: "Gestern auf dem Parteiaktiv konnte jeder sagen[,] was er wollte, aber heute bestimme ich[,] und die Genossen werden doch Offizier. "Informationsbericht über den Stand der Durchführung der Beschlüsse des V. Parteitages der SED in der NVA, 1958, SAPMOBArch, DY 30/IV 2/12/10, B1. 255.

14 Über die Rolle der Partei in der NVA. Protokoll Nr. 4/58 der Sitzung des Politbüros des ZK der SED, 14. Januar 1958, SAPMO-BArch, DY 30/J IV 2/2/576, Bl. 192.

15 Plan zur Auswertung und Durchführung des Beschlusses des Politbüros vom 14. Januar 1958 „Über die Rolle der Partei in der NVA“, 1. Februar 1958, BA-MA, VA-P-01/7535, Bl. $135 \mathrm{f}$.

16 Über die Rolle der Partei in der NVA. Protokoll Nr. 4/58 der Sitzung des Politbüros des ZK der SED, 14. Januar 1958, SAPMO-BArch, DY 30/J IV 2/2/576, Bl. 192.

17 Ebenda.

18 Ebenda.

19 Bericht über die Durchführung der Berichtswahlversammlungen und der bis zum 10. Mai 1958 abgeschlossenen Delegiertenkonferenzen in der NVA, 1958, SAPMO-BArch, DY 30/IV 2/12/10, Bl. 49. 
sche Erwägungen dabei in den Hintergrund. So äußerten Offiziere immer wieder, sie hätten in Anbetracht der „Vielzahl der militärischen Aufgaben keine Zeit für die politische Arbeit“. Diese betrachteten sie vor allem „als Angelegenheit der Politorgane" 20 . Offensichtlich lehnte eine große Zahl von Offizieren die starke Stellung der Partei in der NVA und ihre Eingriffe in die militärischen Entscheidungsprozesse ab. Äußerungen wie der Satz „wozu brauchen wir eine Partei, wenn wir unseren Dienst gut durchführen[?]" beweisen ${ }^{21}$, daß im Offizierskorps von Beginn an ein deutlicher Wunsch nach militärischer Autonomie existierte.

Besonders die Offiziere der Truppenführung wollten militärische Fragen ausschließlich auf der Basis fachlicher Kriterien entscheiden. Eine Politisierung militärischer Entschlüsse und die extensive Beteiligung des Parteiapparates daran sah man als nicht zweckmäßig an. Es bestand die grundsätzliche Tendenz der militärischen Führungsgremien, die Parteiorgane nach Möglichkeit gezielt zu ignorieren, da deren Mitsprache die Entscheidungsprozesse überfrachtete, schwerfällig und somit ineffizient werden ließ. Aufgrund der Tatsache, daß ihre Existenz im Rahmen des militärischen Organisationszusammenhanges sachlogisch überflüssig war, wurden sie andererseits häufig auch unbewußt übergangen. Vor diesem Verhalten waren scheinbar auch hohe Parteifunktionäre in militärischen Führungspositionen nicht gefeit. So beklagte die ZK-Abteilung für Sicherheitsfragen 1958, der damalige 1. Stellvertreter des Ministers für Nationale Verteidigung Hoffmann habe "einfach" die Versetzung eines Feldwebels in sein Sekretariat befohlen, ohne darüber die Parteileitung seiner Grundorganisation sowie die des Ministeriums in Kenntnis zu setzen, „geschweige mit diesen zu beraten“22.

Die SED griff die ablehnende Haltung von Seiten des Offizierskorps gegenüber der umfassenden politischen Durchdringung des Militärs als sogenannten „Nurfachmannstandpunkt“ an. Dieser führe „unvermeidlich zum bürgerlichen Objektivismus, ... zur Unparteilichkeit ... und zur Erhebung der persönlichen Interessen über die der Gesellschaft". Die im Grundsatz sachlich motivierte Forderung nach militärischer Autonomie wurde von der SED politisch diffamiert: Für sie stützte „der Nurfachmann“ mit seinem Verhalten „objektiv den Klassengegner“. Das bedeute „letzten Endes die Ablehnung der führenden Rolle der Partei“23.

Vor allem fürchtete die SED nach 1956, daß diese Tendenz durch das Wirken der ehemaligen Wehrmachtoffiziere in der NVA befördert werden könnte. Man sah die Gefahr, daß gerade diese Offiziere, die wegen ihres militärischen Sachverstandes vor allem auch in Lehreinrichtungen eingesetzt waren, dem Offiziersnachwuchs das traditionelle Leitbild eines Offiziers vermitteln würden. Unmittelbar vor Gründung der NVA hatte die Hauptabteilung I des MfS bemerkt: „Speziell bei ehemaligen Angehörigen der faschistischen Wehrmacht zeigten sich Erscheinungen der Verherrlichung und Popularisierung der Traditionen der faschistischen Wehrmacht

20 Entwurf des Referats des Ministers für Nationale Verteidigung für die Eggersdorfer Tagung, 1957, SAPMO-BArch, DY 30/IV 2/12/10, Bl. 5.

21 Bericht über den politisch-moralischen Zustand in den Verbänden, Truppenteilen und Dienststellen der NVA. Protokoll Nr. $3 / 58$ über die Sitzung des Kollegiums des Ministeriums für Nationale Verteidigung, 23. April 1958, BA-MA, AZN/29178, Bl. 148.

22 Die politische, militärische und fachliche Ausbildung sowie die Qualifizierung durch Fernstudium, 1958, SAPMO-BArch, DY 30/IV 2/12/32, Bl. 45.

23 Clarius, Über die politische Erziehung der Offiziere, S. 2. 
(besonders Luftwaffe, Kriegsmarine und Waffen-SS) und das Bestreben, diese Traditionen am Leben zu erhalten."24 Diese sogenannten „Schlacken der Vergangenheit" 25 waren ein entscheidender Grund dafür, warum die ehemaligen Wehrmachtsoffiziere aus dem Dienst ausscheiden mußten, sobald adäquater Ersatz zu Verfügung stand. Als Beispiel für die divergierenden Ansichten in vielen Fragen führte Hoffmann rückblickend an, für die SED-Funktionäre in der militärischen Führung sei jeder Befehl „zuallererst eine politische Entscheidung“ gewesen. Generalleutnant Vincenz Müller ${ }^{26}$ etwa habe dagegen die Auffassung vertreten: „Ein Befehl wird erteilt, klar und deutlich, er wird vom Unterstellten wiederholt, und damit hat sich's!“27

Wenn es der SED nach 1957 zunehmend gelang, die Autonomisierungstendenzen innerhalb des Offizierskorps zurückzudrängen, so lag dies vor allem daran, daß den Offizieren bei nicht parteikonformem Verhalten neben einer Parteistrafe zugleich auch dienstrechtliche Sanktionen drohten. Im äußersten Fall bedeutete dies die Entlassung aus der $\mathrm{NVA}^{28}$ und damit den Entzug einer gesicherten und gutdotierten beruflichen Existenz. In Anbetracht des politischen Drucks und der nicht kalkulierbaren, häufig willkürlichen Strafen verfielen leitende Offiziere seit 1958 daher in ihrer Führungstätigkeit immer wieder auch in das andere Extrem, indem sie bestrebt waren, „jegliche Entscheidungen grundsätzlich durch kollektive Beratungen herbeizuführen" 29 .

Doch dieses Verhalten wurde von Seiten der SED ebenso kritisiert. Es hieß, die Kommandeure versuchten, sich „hinter der Meinung der Parteileitung zu verstekken" ${ }^{30}$. Hoffmann bemerkte, man müsse den Eindruck gewinnen, daß die Offiziere Angst hätten, „klare Befehle zu geben“ und diese durchzusetzen ${ }^{31}$. Dölling seinerseits spitzte diese Sichtweise weiter $\mathrm{zu}$, indem er hinter diesem Verhalten letztlich eine politische Motivation vermutete. Er fragte, vor wem die Offiziere Angst haben sollten: „Vor der Partei? ... 91\% unserer Offiziere sind Parteimitglieder, was wären das für Parteimitglieder, die Angst haben vor ihrer eigenen Partei? Ich glaube, wenn eine solche Stimmung da ist, daß man sich nicht getraut [,] auf der Grundlage kollektiver Beratungen ... exakte Befehle zu geben, ... dann ist das nicht einfach ein organisatorisches Problem, sondern hängt damit zusammen, daß ein Teil der Offiziere politisch mit der Partei nicht übereinstimmt." ${ }^{\text {"32 }}$

24 Chronik der Hauptabteilung I, BStU, ZA, MfS HA I 95, Bl. 134.

25 Einschätzung der leitenden Kader der Militärakademie „Friedrich Engels“, 1961, SAPMOBArch, DY 30/IV 2/12/29, Bl. 233.

26 Müller war schon in der Wehrmacht Generalleutnant gewesen. 1953-1955 war er Chef des Hauptstabes der KVP; denselben Posten bekleidete er bis 1958 auch in der NVA.

27 Hoff́mann, Moskau - Berlin, S. 313.

28 Vgl. Haueis, Die führende Rolle der SED in der Nationalen Volksarmee, S. $435 \mathrm{f}$.

29 Protokoll der 2. Tagung der II. Delegiertenkonferenz der Parteiorganisationen der SED in der NVA, 25./26. März 1959, BA-MA, VA-P-01/556, Bl. 76.

30 Einleitung für die Aussprache und Beratung mit den Parteisekretären zu dem Thema: „Die Ergebnisse bei der Erfüllung des Beschlusses des Politbüros vom 14. Januar 1958 auf dem Gebiet der Verwirklichung der führenden Rolle der Partei in den Einheiten und Truppenteilen des MB III“, 1958, SAPMO-BArch, DY 30/IV 2/12/9, Bl. 126.

31 Protokoll der 2. Tagung der II. Delegiertenkonferenz der Parteiorganisationen der SED in der NVA, 25./26. März 1959, BA-MA, VA-P-01/556, Bl. 247.

32 Ebenda, B1. 300. 
Symptomatisch für die Reaktionen der militärischen Vorgesetzten auf die politische Indoktrination war jedoch nicht nur ein Mangel an Eigeninitiative in der Führungstätigkeit. Viele Offiziere entwickelten aus Mangel an Vertrauen in eine sachliche Bewertung ihrer Arbeit auch einen außerordentlich autoritären Führungsstil, um so ihre Autorität gegenüber den Unterstellten zu behaupten und jede Diskussion von vornherein zu unterbinden. Dieses Verhalten wurde häufig noch dadurch befördert, daß viele junge Offiziere aufgrund der verfehlten Personalpolitik in den fünfziger Jahren mit den Aufgaben eines militärischen Vorgesetzten überfordert waren. Tatsächlich machte die Entwicklung spezifisch sozialistischer Beziehungen zwischen Offizieren und Soldaten in der NVA kaum Fortschritte. Bereits 1956 hatte man feststellen müssen, daß Offiziere ihren Untergebenen gegenüber oft „überheblich und arrogant" auftraten. Auch seien sie durch die Vorgesetzten „beleidigt, ungerecht bestraft, mit gerichtlichen Strafen bedroht und sogar geschlagen" worden ${ }^{33} .1959$ hatte sich diese Situation nur unwesentlich geändert. Immer noch gab es Fälle, bei denen die „Menschenwürde“ und die „Rechte“ von Soldaten "gröblichst durch Offiziere verletzt" wurden ${ }^{34}$.

Es war nicht zuletzt eine pädagogische Zielsetzung des Produktionseinsatzes von Offizieren und ihres Dienstes als Soldat in der Truppe, diese Auswüchse zu beseitigen und das dienstliche Verhältnis zwischen Vorgesetzten und Unterstellten nachhaltig zu verbessern. Die Erziehungsmaßnahmen stießen jedoch bei einem Teil des Offizierskorps auf massive Ablehnung.

Als Reaktion auf die vom V. Parteitag der SED 1958 beschlossene zeitweilige Tätigkeit in der Produktion äußerte beispielsweise ein Leutnant der 4. Flottille, er habe „keine Lust mehr" und wolle nach Hause gehen. Die Direktive gebe ihm den Rest. Ein anderer Leutnant derselben Flottille sagte: „Ich habe mich für die Nationale Volksarmee gemeldet und nicht für die Produktion, wenn ich dahin gehen muß, dann bleibe ich gleich dort." Die Enttäuschung und Verbitterung, die bei vielen Offizieren angesichts dieser Maßnahme geherrscht haben muß, zeigt die Bemerkung eines Leutnants des MSR 29, die Direktive sei der "größte Betrug“. Er befand: „In keinem anderen Staat wird man so betrogen, wie im Arbeiter-und-BauernStaat.“ 35

Ebenso wurde der ZK-Beschluß von 1959 über den Dienst von Offizieren als Soldat in der Truppe von Teilen des Offizierskorps kategorisch abgelehnt. Dazu hieß es unter anderem: „Die Sache wurde von China ohne Beachtung unserer Verhältnisse übernommen." Ein Oberleutnant etwa vertrat die Meinung, daß es Zeit werde, aus der Armee auszuscheiden, wenn jetzt solche Maßnahmen eingeführt würden ${ }^{36}$. Auch mußten bei der SED Zweifel an der internationalistischen Einstel-

33 Bericht über den politisch-moralischen Zustand in den Einheiten der Land-, Luft- und Seestreitkräfte. Protokoll Nr. 7/56 über die Sitzung des Kollegiums des Ministeriums für Nationale Verteidigung, 21. September 1956, BA-MA, VA-01/2030, Bl. 92.

34 Protokoll der 2. Tagung der II. Delegiertenkonferenz der Parteiorganisationen der SED in der NVA, 25./26. März 1959, BA-MA, VA-P-01/556, Bl. 68.

35 Informationsbericht über die politische Arbeit und die Stimmungen der Armeeangehörigen in der ersten Monatshälfte Dezember 1958, 22. Dezember 1958, BA-MA, VA-01/5050, Bl. $143 \mathrm{f}$.

36 Informationsbericht über die Stimmungen und Meinungen der Armeeangehörigen in der ersten Monatshälfte Februar 1959, 19. Februar 1959, BA-MA, VA-01/5050, Bl. 96. 
lung der NVA-Offiziere aufkommen, wenn etwa ein Hauptmann äußerte: „Wenn die Chinesen ihre Offiziere als Soldaten dienen lassen, haben wir das noch lange nicht nötig. Was haben wir denn mit den Chinesen gemeinsam?". Dabei wurde auch deutlich, daß im Offizierskorps entgegen dem von der Partei postulierten sozialistischen Typus noch immer eine ausgesprochen traditionelle Vorstellung vom Leitbild des Offiziers existierte. So äußerte ein Oberleutnant: „Das, was Walter Ulbricht vorgeschlagen hat, ist eines deutschen Offiziers nicht würdig, das kann man bei den Chinesen machen, aber nicht bei uns. " 37

Für die Offiziere der NVA stellte es in der Führungsarbeit offenbar eine große Belastung dar, die primär politisch geprägten Forderungen und Argumentationsweisen der Partei mit den militärischen Organisationsprinzipien und deren Begrifflichkeit zu verbinden. Häufig ließen sich diese beiden Bezugssysteme jedoch nicht vermitteln, da die politischen Kategorien innerhalb des militärischen Systems über keine Anschlußfähigkeit verfügten. Die Vorgesetzten waren insofern mit dem Dilemma konfrontiert, daß parteikonformes Verhalten im Rahmen der Entscheidungsprozesse nicht gleichzeitig auch ein hohes $\mathrm{Maß}$ an Problemlösungskompetenz besaß. Eine im Sinne der Parteidoktrin „richtige“ Argumentation oder Entscheidung war in der Praxis unter Umständen vollkommen ungeeignet, militärische Fragen sachbezogen und effizient zu lösen. So konnte sich etwa die bevorzugte Beförderung politisch zuverlässiger Offiziere negativ auf die militärische Qualität des Leitungspersonals auswirken, da die fachliche Qualifikation im Rahmen der Kaderentscheidung nur eine nachrangige Bedeutung hatte.

Der Zwang, permanent zwei logisch nicht zu verbindende Sichtweisen zu vermitteln und unter diesen Bedingungen schnell Entschlüsse zu fassen, dürfte bei vielen Offizieren einen Zustand tiefer Verunsicherung erzeugt haben. Nicht selten mag diese Situation als schizophren wahrgenommen worden sein. Um dem psychischen Druck zu entgehen, der dadurch auf vielen Vorgesetzten lastete, war es typisch, daß sie ihre Führungstätigkeit aus Angst vor Fehlern auf das Nötigste reduzierten. Der Schriftsteller Günter de Bruyn hat auf der Basis der eigenen Erfahrungen in der DDR im Hinblick auf eine derartige Lage geäußert, daß „die Versuchung“ groß war, „der Vernunft abzuschwören, um ehrlichen Herzens in der Glaubensgemeinschaft aufgehen zu können“. Doch sei auch dieser „Weg ... alles andere ... als bequem" gewesen: „Denn verlangt wurde nicht nur Unterordnung, sondern auch eine Selbstverleugnung, die nicht nur eigenes Denken, sondern auch eigenes Wahrnehmen verbot. Man mußte ... sich blind machen und taub stellen und bei jeder Kursänderung glauben, es gehe geradeaus. “38 Aber selbst das Vertreten eines dezidiert parteilichen Standpunktes schützte die Offiziere nicht in jeder Hinsicht vor Kritik seitens der Partei. Auch eine Position im Sinne der gerade gültigen Parteilinie barg das Risiko, zu einem späteren Zeitpunkt als Beweis für „Abweichlertum“ zitiert zu werden ${ }^{39}$.

Angst, fehlendes Vertrauen in die Vorgesetzten und die Unsicherheit hinsichtlich der Bewertungsmaßstäbe, die an die Führungstätigkeit angelegt wurden, bewirkten

37 Einige Probleme des politisch-moralischen Zustandes der NVA, 4. April 1960, BStU, ZA, MfS ZAIG 260, Bl. 5.

38 De Bruyn, Zwischenbilanz, S. $374 \mathrm{f}$

39 Lolland, Zu Befehl, Genosse Unterleutnant, S. 40. 
bei vielen Offizieren Resignation, menschliche Vereinsamung, innere Emigration, Depressionen oder Aggressionen. Diese psychischen Schädigungen waren entscheidende Gründe für die schlechte Disziplin und den verbreiteten Alkoholismus innerhalb des Offizierskorps ${ }^{40}$.

Die SED perzipierte ein derartiges Verhalten jedoch nicht als Folge der spezifischen Bedingungen, unter denen die Offiziere ihre Tätigkeit ausüben mußten. Threr Auffassung nach fehlte vielen Offizieren vielmehr der „innere Elan“, um mit Begeisterung am Aufbau des Sozialismus teilzunehmen. Einen wesentlichen Grund hierfür sah die Partei in ihrer "sicheren materiellen Lage“. Die Offiziere würden sich daher lethargisch gegenüber den Schwierigkeiten in der Dienstdurchführung verhalten. Es gebe sogar "zahlreiche Beispiele“, daß Offiziere dem Alkohol verfielen und in grober Weise gegen die sozialistische Moral verstießen ${ }^{41}$.

Demzufolge machte die SED auch die Gründe für die hohe Selbstmordrate in der NVA als tragischste Folge des hohen psychischen Drucks, der auf den Offizieren lastete, in erster Linie an persönlichen Problemen fest. „In den wenigsten Fällen“, so hieß es, hätten „die Ursachen“ für die Selbstmorde „in der Dienstdurchführung“ gelegen. Sie seien vielmehr ,in zerrütteten Familienverhältnissen und unmoralischem Lebenswandel zu suchen"42. Dölling kleidete diese Sichtweise zynisch in die Formel, daß diejenigen, die Selbstmord begingen, „falsche Vorstellungen vom Leben" gehabt hätten ${ }^{43}$.

1956 nahmen sich 39 NVA-Angehörige das Leben ${ }^{44} ; 1957$ waren es $42^{45}$ und 1958 insgesamt 3946. In den folgenden Jahren wies die Statistik eine leicht fallende Tendenz auf: 1959 begingen 36 Armeeangehörige Selbstmord, darunter waren 9 Offi-

$40 \mathrm{Vgl}$. Analyse über die in der Zeit vom 1. Januar bis 31. Dezember 1959 bearbeiteten Strafverfahren gegen Offiziere der NVA, 2. Februar 1960, BA-MA, VA-01/13496, Bl. 9. Bei jungen Offizieren lag ein derartiges Verhalten unter anderem auch darin begründet, daß nach Gründung der NVA eine Reihe von ihnen im Alter zwischen 18 und 22 Jahren im Rahmen von Partei- oder Verbandsaufträgen häufig unfreiwillig - in die Streitkräfte delegiert und zu Offizieren ausgebildet worden waren. Viele waren daher schlecht motiviert und verweigerten sich, weil sie sich nicht wirklich mit ihrem Beruf identifizierten. Wegen des Offiziersmangels in der NVA konnten sie jedoch selten vorzeitig aus dem Dienst ausscheiden. Buddrus, „Kaderschmiede für den Führungsnachwuchs"?, S. 177.

41 Entwurf des Referats des Ministers für Nationale Verteidigung für die Eggersdorfer Tagung, 1957, SAPMO-BArch, DY 30/IV 2/12/10, Bl. 24.

42 Statistischer Bericht über besondere Vorkommnisse für das II. Quartal 1957, 15. Juli 1957, BA-MA, VA-01/5961, Bl. 118.

43 Protokoll der 2. Tagung der II. Delegiertenkonferenz der Parteiorganisationen der SED in der NVA, 25./26. März 1959, BA-MA, VA-P-01/556, Bl. 39.

44 Bericht Nr. 2/58 über den Stand der Einsatzbereitschaft, der operativen, militärischen und politischen Ausbildung der NVA der DDR nach dem Stand vom 1. Januar 1958, BA-MA, VA-01/4354, Bl. 10. Für die Jahre 1956 bis 1958 liegen - soweit nach der Quellenrecherche ersichtlich - keine konkreten Zahlen über Selbstmorde von Offizieren vor.

45 Bericht Nr. 2/58 über den Stand der Einsatzbereitschaft, der operativen, militärischen und politischen Ausbildung der NVA der DDR nach dem Stand vom 1. Januar 1958, BA-MA, VA-01/4354, Bl. 10.

46 Bericht Nr. 4/59 über den Stand der Einsatzbereitschaft, der operativen, militärischen und politischen Ausbildung der NVA der DDR nach dem Stand vom 1. Januar 1959, BA-MA, VA-01/4354, Bl. 151. 
ziere $^{47} .1960$ gab es insgesamt 25 Suizide einschließlich 6 von Offizieren ${ }^{48}$. Bis zum Mauerbau am 13. August 1961 verzeichnet die Statistik weitere 17 Selbstmorde; unter den Toten waren wiederum 4 Offiziere ${ }^{49}$.

Ulbricht hielt die Selbstmordzahlen für „unerhört hoch“. Dafür gebe es „keine Entschuldigung“. Er fragte sich, was das für ein Zustand sei, wenn sich Soldaten oder Offiziere erschießen würden, weil sie „Frauengeschichten“ hätten. Auf der Eggersdorfer Tagung sagte er: „Kann man nicht die Dinge in Ordnung bringen? ... Selbstverständlich muß man ihnen helfen." Es gebe doch keinen Menschen, der sein "ganzes Leben Engel gewesen" sei ${ }^{50}$. Offenbar fehlte aber vielen Offizieren das Vertrauen sowohl in einen fairen Umgang mit den individuellen Fehlern und Vergehen als auch in eine angemessene Hilfe von Seiten der Vorgesetzten und der Partei. Immer wieder scheint daher der Selbstmord als einziger Ausweg aus einer Zwangslage angesehen worden zu sein.

Eine andere Reaktion auf die politische Durchdringung der militärischen Sphäre war der Rückzug in das Privatleben. Viele Offiziere versuchten, außerhalb der Armee der Politisierung durch die SED zu entgehen, zumal es keine korporative Identität gab, die ein Gegengewicht zur Parteidoktrin hätte bilden können. Dieses Verhalten war charakteristisch für die DDR als „Nischengesellschaft“. In der privaten Sphäre versuchte der einzelne, „Freiräume von der herrschenden Lehre“ zu finden ${ }^{51}$. Vor allem in den fünfziger Jahren hielt ein Teil der Offiziere offenbar noch an tradierten Werten und Verhaltensmustern fest. Insbesondere die Familie fungierte dabei als Rückzugsraum. Nur hier war es noch möglich, die individuellen Normen des Zusammenlebens selbst zu bestimmen, während die Partei die allgemeinen Moralvorstellungen bereits in ihrem Sinne fixiert hatte ${ }^{52}$. Stoph kritisierte in diesem Zusammenhang, bei den Offizieren finde man „sehr häufig kleinbürgerliche Erscheinungen in ihrem Familienleben"53.

Gerade in den ersten Jahren nach Gründung der NVA scheint sich eine Reihe Offiziere trotz der Indoktrinierung der SED geweigert zu haben, ihre Beziehungen zur Kirche und die Ausübung ihres christlichen Glaubens gänzlich aufzugeben. Bei Gründung der NVA lag der Anteil allein von Mitgliedern und Kandidaten der SED, die gleichzeitig Mitglieder der Kirche waren, in norddeutschen Einheiten teilweise

47 Einige Probleme des politisch-moralischen Zustandes der NVA, 4. April 1960, BStU, ZA, ZAIG 260, Bl. 10.

48 Bericht über einige Schwächen innerhalb der NVA, 8. April 1961, BStU, ZA, ZAIG 365, Bl. 15.

49 Bericht über einige Probleme zur Einschätzung des politisch-moralischen Zustandes der bewaffneten Kräfte der DDR, 2. Oktober 1961, BStU, ZA, ZAIG 479, Bl. 7. Die Selbstmordrate in der NVA lag während dieses Zeitraums rund anderthalb- bis zweieinhalbmal über der der Bundeswehr. Dort nahmen sich 1957-1961 auf 100000 Mann gesehen pro Jahr durchschnittlich 17,5 Soldaten das Leben. Preuschoff, Suizidales Verhalten in deutschen Streitkräften, S. 253.

50 Protokoll der Eggersdorfer Tagung, 12./13. Juni 1957, BA-MA, VA-P-01/037, Bl. 148.

51 Gaus, Wo Deutschland liegt, S. $164 \mathrm{f}$.

$52 \mathrm{Vgl}$. Die 10 Gebote des sozialistischen Menschen, S. 1.

53 Er konstatierte ferner: „Viele Frauen, besonders auch von Offizieren, nehmen wenig oder keinen Anteil am gesellschaftlichen Leben, benehmen sich gegenüber den Werktätigen überheblich und vertreten nicht die Interessen unseres Staates in der Öffentlichkeit. "Protokoll der Delegiertenkonferenz der Parteiorganisationen der SED im Dienstbereich des Ministeriums für Nationale Verteidigung, 5.-11. März 1956, BA-MA, VA-P-01/036, Bl. 52. 
immer noch bei rund 70 Prozent ${ }^{54}$. Stoph monierte zu diesem Zeitpunkt: „Es gibt Offiziere, die ihre Untergebenen davon zu überzeugen versuchen, daß es notwendig ist, ihre Kinder an der Jugendweihe teilnehmen zu lassen, ihre eigenen Kinder aber lassen sie konfirmieren." 55

Das Festhalten vor allem junger Offiziere an der kirchlichen Bindung lag wohl auch in der Tatsache begründet, daß der Offiziersnachwuchs der Jahre 1956-1958 zu einem nicht unwesentlichen Teil aus dem ländlich-bäuerlichen Milieu stammte ${ }^{56}$. Der Bezug dieser Offiziere zur Kirche dürfte traditionell ausgeprägter gewesen sein als bei denen, die aus der Arbeiterschaft kamen. Zudem hielten anscheinend insbesondere jüngere Offiziere den Bezug zur Kirche aufrecht, weil sie, sehr zum Mißfallen der SED, häufig Frauen aus bürgerlichen Kreisen geheiratet hatten ${ }^{57}$.

Die ablehnende Haltung gegenüber der politischen Durchdringung des Militärs äußerte sich darüber hinaus auch in einer verbreiteten Distanz zu der von der SED verfolgten offensiven Ausrichtung der NVA. Dementsprechend ist es verständlich, warum Dölling 1959 nachdrücklich forderte, alle „Unklarheiten“ darüber auszuräumen, „daß nicht wir diejenigen sind, die einen Krieg wollen, daß nicht wir mit Raketenwaffen Kassel, Braunschweig, Dortmund oder Köln bedrohen, sondern umgekehrt, die westdeutschen Militaristen die friedliche Aufbauarbeit der Deutschen Demokratischen Republik vernichten wollen "58. Die operativen Planungen und die Manövertätigkeit gaben den Armeeangehörigen hierfür offenbar jedoch wenig Anlaß. So mußte Hoffmann einräumen, daß etwa die zweiseitige Truppenübung, die im März 1959 im Militärbezirk III stattgefunden hatte, in einen Zusammenhang gebracht wurde "mit der Lösung der Berlin-Frage“59.

Die SED-Führung sah sich mit der Lage konfrontiert, daß ein Teil der Offiziere und sogar auch der Kommandeure von der „Gefährlichkeit des aggressiven westdeutschen Militarismus" nicht überzeugt war60. Dies war nach Ansicht der SED ein entscheidender Grund dafür, daß verbreitet Unklarheiten über die „wirklichen Aufgaben" der NVA bestanden. Ein Mitarbeiter der Sicherheitskommission äußerte dazu im März 1960, es gebe „eine Art Verteidigungskomplex“ in der Truppe ${ }^{61}$ : „So ist es z.B. vielen Offizieren selbst auch noch nicht klar, daß wir den Gegner nicht nur zurückdrängen werden auf sein Territorium, sondern daß wir ihn restlos ver-

54 Ebenda, Bl. 304.

55 Ebenda, Bl. 52.

56 Für viele der Offiziere, die aus dem ländlichen Umfeld stammten, war der Offiziersdienst in erster Linie eine berufliche Durchgangsstation, die ihnen später einen leichteren Zugang zu städtisch-industriellen Berufen, aber auch einen sozialen Aufstieg ermöglichen sollte. Vgl. Auerbach, Der Stellenwert der Nationalen Volksarmee, S. $69 \mathrm{ff}$.

57 Protokoll der Delegiertenkonferenz der Parteiorganisationen der SED im Dienstbereich des Ministeriums für Nationale Verteidigung, 5.-11. März 1956, BA-MA, VA-P-01/036, Bl. 303.

58 Protokoll der 2. Tagung der II. Delegiertenkonferenz der Parteiorganisationen der SED in der NVA, 25./26. März 1959, BA-MA, VA-P-01/556, Bl. 21.

59 Ebenda, Bl. 243.

60 Auskunftsbericht über den Stand der Einsatzbereitschaft der NVA. Protokoll der 19. Sitzung der Sicherheitskommission des ZK der SED, 9. Januar 1958, BA-MA, DVW 1/39561, Bl. 32.

61 Protokoll der Arbeitsberatung des Ministers für Nationale Verteidigung mit den Chefs der Verwaltungen und Abteilungsleitern des Ministeriums für Nationale Verteidigung, 23. März 1960, BA-MA, VA-01/6106, Bl. 50. 
nichten müssen." ${ }^{62}$ Die ZK-Abteilung für Sicherheitsfragen konstatierte zur gleichen Zeit, die Mehrheit der Armeeangehörigen habe noch keine Vorstellungen darüber, „dass sie im Falle eines Krieges bei der Erfüllung ihrer Aufgaben auf westlichem Territorium als Befreier der Arbeiter, Bauern und aller patriotischen Kräfte unseres Volkes" handelten ${ }^{63}$. Diese Einschätzungen zeigen, daß außer zur Verteidigung des eigenen Territoriums ein möglicher Einsatz der Streitkräfte - etwa über die Grenzen der DDR hinweg - in der NVA nur bedingt als legitim angesehen wurde. Überhaupt scheint es bei einigen Armeeangehörigen in Anbetracht eines drohenden nuklearen Konfliktes zwischen NATO und Warschauer Pakt grundsätzliche Zweifel am Sinn eines militärischen Einsatzes gegeben zu haben. Stoph äußerte 1956: „Es gibt solche Meinungen, das[s] dort, wo die Atombombe einschlägt [,] alles verloren ist." 64

Die Kritik an der SED kam zum Teil auch in politischem Widerspruch zum Ausdruck. So wurden etwa die "Oder-Neiße-Grenze" und die "deutsch-polnische Freundschaft" in Zweifel gezogen. Ebenso gab es negative Äußerungen zum „Eingreifen der Sowjetarmee in Ungarn" und bezüglich der unzureichenden Informationen in der Presse und im Rundfunk darüber. Die schlechte Nachrichtenlage in der NVA wurde offenbar gerade von Offizieren immer wieder zum Anlaß genommen, westliche Radiosender abzuhören ${ }^{65}$. Zudem signalisierte eine Reihe von Armeeangehörigen fehlendes Vertrauen in die Geschlossenheit des sozialistischen Lagers und die "Festigkeit des Bestandes der Arbeiter- und Bauern-Macht" in der DDR ${ }^{66}$. Dabei scheint die Überzeugung verbreitet gewesen zu sein, daß „der Aufbau des Sozialismus in der DDR ... nur die demokratische Wiedervereinigung" verhindere ${ }^{67}$. Ferner wurde die Auffassung vertreten, in „imp. Armeen" würden „die Soldaten mehr Freiheit besitzen“, und es gab Forderungen nach „Zulassung der CDU" (Christlich-Demokratische Union) in der NVA68.

Hinter derartigen Auffassungen stand keineswegs in jedem Fall ein klares politisches Konzept. Oft waren sie nur Anzeichen einer Protesthaltung, die sich an bestimmten Maßnahmen der Partei oder an der zunehmenden politischen Durchdringung der gesamten militärischen Sphäre entzündete. Zum Teil wurde der Protest dabei auch durch nazistische Äußerungen artikuliert; ein Phänomen, das sich in der NVA offenbar in den folgenden Jahrzehnten verstärkte ${ }^{69}$. So gab es in den Unter-

62 Ebenda, Bl. 45.

63 Erfahrungen und Schlußfolgerungen aus dem Brigadeeinsatz des ZK der SED im Mot. Schützenregiment 2, Mot. Schützenregiment 3, Panzerregiment 1 und Stab der 1. Mot. Schützendivision, 1960, SAPMO-BArch, DY 30/IV 2/12/22, Bl. 10.

64 Protokoll Nr. $4 / 56$ über die Sitzung des Kollegiums des Ministeriums für Nationale Verteidigung, 30. Mai 1956, BA-MA, VA-01/2029, Bl. 11.

65 Einschätzung des politisch-moralischen Zustandes und der Stimmungen der Verbände und Truppenteile, 1956, BA-MA, VA-P-01/1752, Bl. 91.

66 Ebenda.

67 Bericht über den Stand des politischen Bewußtseins und die Moral in der NVA. Protokoll Nr. 3/57 über die Sitzung des Kollegiums des Ministeriums für Nationale Verteidigung, 6. Mai 1957, BA-MA, VA-01/2032, Bl. 41.

68 Die Abwehrarbeit des MfS in den bewaffneten Formationen der DDR. Schulungsmaterial, Dezember 1958, BStU, ZA, JHS Sicherheitsfilm Z. 214/58, Bl. 34.

69 Vgl. für die Zeit nach 1965 Eisenfeld, Nazistischer Geist im sozialistischen Waffenrock, S. 14. 
künften immer wieder „Hakenkreuzschmierereien"70. Ferner wurden etwa $1958 \mathrm{im}$ Nachrichten-Bataillon des Militärbezirkes III drei Soldaten verhaftet, weil sie unter anderem Hitler verherrlicht und das „Horst-Wessel-Lied“ gesungen hatten. Der Chef der Hauptabteilung I des MfS Kleinjung machte in diesem Zusammenhang ausdrücklich auf den Umstand aufmerksam, daß weder der Kommandeur noch der Politstellvertreter auf Hinweise reagiert hätten, „dieser Ideologie entgegenzutreten, sie zu zerschlagen" 71 .

Handelte es sich hierbei zumeist um vereinzelt auftretende, nicht organisierte Meinungsäußerungen, so mußte das Verhalten der NVA-Offiziere an den Militärakademien der UdSSR auf die Parteiführung weitaus alarmierender gewirkt haben. 1957 stellte eine eigens eingerichtete Untersuchungskommission fest, daß die „politische Festigkeit in der Mehrheit der deutschen Offiziersgruppen unbefriedigend“ sei $^{72}$. So habe es etwa an der Frunse-Akademie in Moskau ,jahrelang falsche und feindliche Auffassungen gegenüber der Sowjetunion" gegeben ${ }^{73}$. Diese hätten sich beispielsweise darin geäußert, daß ein Oberleutnant der NVA sowjetische Bürger „mit ,Bolschewistenschwein“" beschimpft habe ${ }^{74}$.

Formen des Protestes oder der Verweigerung, wie etwa der Versuch von Offizieren, sich der SED-Mitgliedschaft zu entziehen 75 , ging vermutlich in der Regel - bewußt oder unbewußt - ein längerer persönlicher Meinungsbildungsprozeß voraus. Denn der einzelne dürfte sich über die zu erwartenden Sanktionen der Partei von vornherein im klaren gewesen sein. Letztlich folgte derartigem Verhalten nicht selten eine Desertion, die in der Regel gleichbedeutend mit der Flucht in den Westen war ${ }^{76}$. Die hohe Zahl von Desertionen in der NVA ${ }^{77}$ stellte wegen des Personalmangels in der Zeit bis zum Mauerbau 1961 ein ernstes Problem dar. Zudem wirkten sich die Fahnenfluchten äußerst negativ auf die innere Stabilität der Streitkräfte aus.

Im ersten Jahr des Bestehens der NVA desertierten 233 Armeeangehörige ${ }^{78}$, dar-

70 Bericht über einige Probleme zur Einschätzung des politisch-moralischen Zustandes der bewaffneten Kräfte der DDR, 2. Oktober 1961, BStU, ZA, MfS ZAIG 479, Bl. 6.

71 Bericht über den politisch-moralischen Zustand in den Verbänden, Truppenteilen und Dienststellen der NVA. Protokoll Nr. $3 / 58$ über die Sitzung des Kollegiums des Ministeriums für Nationale Verteidigung, 23. April 1958, BA-MA, AZN/29178, Bl. 167.

72 Bericht der Kommission zur Überprüfung in den Offiziersgruppen der NVA an den Militärakademien in der Sowjetunion. Protokoll der 21. Sitzung der Sicherheitskommission des ZK der SED, 10. April 1958, BA-MA, DVW 1/39563, Bl. 26

73 Ebenda, Bl. 28.

74 Bericht über das Ergebnis der parteimäßigen Überprüfung der besonderen Vorkommnisse im 2. Kurs der Frunse-Akademie (Infanterie), 1. Oktober 1957, SAPMO-BArch, DY 30/IV $2 / 12 / 30, \mathrm{Bl} .7$.

75 So äußerte beispielsweise ein Oberleutnant: „Wenn der letzte Soldat meiner Kp. in die Partei eingetreten ist, ist es für mich immer noch Zeit, Kandidat der SED zu werden. “ Bericht über die Stimmungen der Armeeangehörigen und Erscheinungen in der politischen Arbeit in der ersten Monatshälfte April 1958, BA-MA, VA-01/1752, Bl. 66.

76 Godau, Verführter Verführer. „Ich war Politoffizier der NVA“.

$77 \mathrm{Vgl}$. Wenzke, Fahnenflucht in den Streitkräften der DDR, S. 266 ff.

78 Bericht Nr. 2/58 über den Stand der Einsatzbereitschaft, der operativen, militärischen und politischen Ausbildung der NVA der DDR nach dem Stand vom 1. Januar 1958, BA-MA, VA-01/4354, Bl. 10. Die Desertionszahlen weichen in den Statistiken zum Teil voneinander ab. Der Grund hierfür dürfte die Unterscheidung in versuchte und vollendete Fahnenfluchten sein, die aus den Akten jedoch nicht immer eindeutig hervorgeht. Die angeführten Zah- 
unter 13 Offiziere ${ }^{79} .1957$ lag die Zahl insgesamt bei $180^{80}$ einschließlich 18 Offizieren $^{81} .1958$ waren es 112 Armeeangehörige, darunter 13 Offiziere ${ }^{82}$, im folgenden Jahr gab es insgesamt 71 Deserteure, darunter 11 Offiziere ${ }^{83} .1960$ stiegen die Zahlen offensichtlich wieder an. Unter den 133 Fahnenflüchtigen befanden sich 18 Offiziere ${ }^{84}$. Bis zum Mauerbau am 13. August 1961 desertierten dann noch einmal 62 Angehörige der NVA einschließlich 3 Offizieren ${ }^{85}$.

Bei der überwiegenden Zahl der zwischen 1956 und 1961 desertierten Offiziere handelte es sich um Leutnantsdienstgrade ${ }^{86}$. Der dienstgradhöchste Deserteur während dieses Zeitraums war vermutlich Oberstleutnant Siegfried Dombrowski. Der damalige Stellvertreter des Chefs der „Verwaltung für Koordinierung“ (VfK) der NVA, die die Militärspionage gegen die Bundesrepublik betrieb, setzte sich im August 1958 gemeinsam mit Ehefrau und Kindern in den Westen $a b^{87}$.

Die Desertionen waren jedoch vermutlich in der Mehrzahl der Fälle nicht primär politisch motiviert. Die meisten Offiziere stimmten wohl zumindest grundsätzlich mit den propagierten politischen Zielen der SED überein. Vielmehr waren die Desertionen, denen auch rein persönliche Motive zugrunde lagen, nicht zuletzt eine Folge des allgemein angespannten, teilweise desolaten inneren Zustandes der NVA. Dieser jedoch war zu einem großen Teil das Ergebnis der politischen Durchdrin-

len beziehen sich auf vollendete Desertionen, wobei der niedrigere bzw. am häufigsten in den Quellen genannte Wert berücksichtigt worden ist.

79 Statistische Berichte über Fahnenfluchten und besondere Vorkommnisse, 1957-1959, BA-MA, VA-01/5961, Bl. 18.

80 Bericht Nr. 2/58 über den Stand der Einsatzbereitschaft, der operativen, militärischen und politischen Ausbildung der NVA der DDR nach dem Stand vom 1. Januar 1958, BA-MA, VA-01/4354, Bl. 10.

81 Statistische Berichte über Fahnenfluchten und besondere Vorkommnisse, 1957-1959, BA-MA, VA-01/5961, Bl. 18.

82 Bericht Nr. 6/60 über den Stand der Einsatzbereitschaft, der politischen, operativen und Gefechtsausbildung der NVA der DDR nach dem Stand vom 1. Januar 1960, 28. Januar 1960, BA-MA, VA-01/6247, Bl. 10.

83 Einige Probleme des politisch-moralischen Zustandes der NVA, 4, April 1960, BStU, ZA, MfS ZAIG 260, Bl. 8.

84 Analyse der Fahnen- und Republikfluchten in der NVA im Jahre 1960, SAPMO-BArch, DY 30/IV 2/12/47, Bl. 150.

85 Bericht über einige Probleme zur Einschätzung des politisch-moralischen Zustandes der bewaffneten Kräfte der DDR, 2. Oktober 1961, BStU, ZA, MfS ZAIG 479, Bl. 7.

86 Vgl. Übersicht über Desertionen, 1. Januar - 31. Dezember 1958, BA-MA, VA-01/21890, Bl. 35.

87 Seine Flucht traf die NVA schwer. Durch ihn gewann der Westen Informationen vor allem über Aufgaben, Arbeitsweise und Personal dieser Verwaltung. Als Folge wurden unter anderem rund 200 Offiziere und Soldaten der VfK aus dem Armeedienst entlassen. Zudem konnte die Verwaltung offenbar erst nach fünf Jahren wieder aktiv werden. Zolling/Höhne, Pullach intern, S. 102. Das MfS bearbeitete diese Desertion im Rahmen eines operativen Vorganges. Ziel war entweder die „Rückführung des Dombrowski auf das Territorium der DDR mit dem Ziel seiner Aburteilung" oder die "Liquidierung des Dombrowski an seinem Aufenthaltsort unter der Bedingung, daß ein solches Ereignis von der Presse aufgefangen und publiziert werden" konnte. Sachstandsbericht, 19. Februar 1959, BStU, ZA, MfS AOP 12437/78, Bd. 7, Bl. 37. „Am 20. 6. 1977 erlag Dombrowski während einer Autofahrt von München nach Nürnberg in der Nähe von Ingolstadt einen [sic!] Herzinfarkt, an dessen Folgen er verstarb.“ Abschlußbericht zum Operativ-Vorgang „Doppelgänger“, 19. Juli 1958, $\mathrm{BStU}, \mathrm{ZA}$, MfS AOP 12437/78, Bd. 7, Bl. 108. Eine Verantwortung des MfS für seinen Tod geht aus den Quellen aber nicht hervor. 
gung des Militärs durch die SED. Einige Offiziere sahen sich daher veranlaßt, sich dem daraus resultierenden politischen Druck und den psychischen Belastungen durch Flucht zu entziehen. Insofern entsprachen die wenigsten der desertierten Offiziere und Soldaten der NVA der im Westen vorherrschenden Sicht von einem „überzeugten Antikommunisten“. Anstatt ein „,politisches' Fluchtmotiv“ zu besitzen, wie man es von einem "verfolgten Widerstandskämpfer" hätte erwarten können, äußerten die meisten, sie seien „mit den Zuständen in der DDR ,allgemein unzufrieden "“ gewesen 88 .

Die SED war jedoch offenbar nicht dazu in der Lage, sich ein differenziertes Bild von den verschiedenen Gründen der Fahnenfluchten ebenso wie von anderen nicht systemkonformen Verhaltensweisen zu verschaffen. Entsprechend ihrem ideologischen Erklärungsmuster bewertete sie die Ursachen nach einem simplifizierenden Freund-Feind-Schema. Nach Ansicht der SED waren etwa „kleinliche Augenblicksüberlegungen wie z.B. persönliche Verärgerung, scheinbare Ausweglosigkeit aus familiären und persönlichen Schwierigkeiten, Angst vor Strafe bei begangenen Delikten, ungenügende Bindung zum Kollektiv und zu den Vorgesetzten oder auch offene Abwerbung und Beeinflussung durch den Klassengegner" nur der „äußere Anlaß" für Desertionen oder andere Vergehen ${ }^{89}$. Als eigentliche Ursache, so führte Dölling aus, galt jedoch die „mangelnde Überzeugung von der Gesetzmäßigkeit des Sieges des Sozialismus in ganz Deutschland“. Sie sei „letzten Endes die ideologische Wurzel der meisten Fälle von Fahnenflucht und Fahnenfluchtversuchen", soweit man es nicht "mit direkten Agenten und Spionen zu tun" habe ${ }^{90}$. Die Partei betrachtete letztlich jeden Deserteur als einen Regimegegner. Es muß daher ein alarmierendes Zeichen für die politische Zuverlässigkeit der NVA gewesen sein, wenn man feststellte, eine Desertion werde von der "Mehrheit der Armeeangehörigen“ noch nicht „als ein schändlicher Verrat an der Arbeiterklasse, ein Verbrechen an der DDR und ein Überlaufen zum Klassenfeind betrachtet" $"$.

Es stellt sich die Frage, ob bestimmte Reaktionen von Offizieren auf die politische Durchdringung der NVA als Widerstand zu qualifizieren sind. Grundsätzlich kann man von Widerstand nur dann sprechen, wenn der „Widerstandleistende die Gefahr einer Benachteiligung oder Bestrafung bewußt in Kauf nimmt und sich in seiner Weigerung, Befehle, Anweisungen oder Gesetze zu befolgen, mit Gründen auf sein Gewissen oder ein höheres Recht berufen kann". In Anbetracht dieser Definition unterscheidet sich Widerstand folglich „von der bloßen Dienstpflichtverletzung, Befehlsverweigerung und Gesetzesübertretung "92.

Zur Veranschaulichung sei hier die Fahnen- und Republikflucht eines Leutnants der 7. Panzerdivision genannt: Dieser hatte sich geweigert, Politunterricht zu geben und SED-Mitglied zu werden; letzteres, da „er sich dann in seiner persönlichen Freiheit eingeengt fühle". Zudem hatte er es aufgrund der Religiosität seines Elternhauses abgelehnt, aus der Kirche auszutreten. Nach der Versetzung in eine andere

${ }^{88}$ Lolland, Zu Befehl, Genosse Unterleutnant, S. 12.

89 Jahresbericht 1957 der Politischen Verwaltung, BA-MA, VA-P-1/030, B1. 8.

90 Protokoll der Delegiertenkonferenz der Parteiorganisationen der SED in der NVA, 24.-25. Juni 1958, BA-MA, VA-P-01/038, Bl. 22

91 Bericht über den Stand der Durchführung der Beschlüsse des V. Parteitages der SED in der NVA, 20. Oktober 1958, SAPMO-BArch, DY 30/IV 2/12/10, Bl. 245.

92 Münkler, Widerstand, S. $790 f$. 
Einheit, die der Leutnant als „Strafversetzung“ empfand, stellte ein Mitglied der Parteileitung ihn ultimativ vor die Wahl, endlich in die SED einzutreten oder aus der Armee entlassen zu werden. Daraufhin entschloß er sich, in die Bundesrepublik zu desertieren ${ }^{93}$.

Eine derartige Desertion ist als Widerstand zu qualifizieren. Der Leutnant konnte sich bei seiner Flucht auf die Einschränkung seiner Gewissens-, Religions- und Meinungsfreiheit berufen. Ein individueller Anspruch auf diese Rechte und Freiheiten ließ sich unter anderem aus den Artikeln 18 und 19 der „Allgemeinen Erklärung der Menschrechte" vom 10. Dezember 1948 herleiten ${ }^{94}$. Das erzwungene Ausscheiden aus der NVA stellte für ihn keine Alternative dar, da dieses zum einen sein Recht auf freie Berufswahl mißachtete und zum anderen eine Gefährdung seiner Existenzgrundlage bedeutete. In dieser Situation erwies sich die Desertion als einzige Handlungsoption. Es ist davon auszugehen, daß sich der Leutnant über die drohenden strafrechtlichen Konsequenzen hinsichtlich seiner Handlung bewußt war. Fahnenflucht war nicht nur gemäß Strafrechtsergänzungsgesetz strafbewehrt, sondern galt im Rahmen der Propaganda der SED auch als „das schwerste und verabscheuungswürdigste Verbrechen gegen die militärische Disziplin"95. Da der Leutnant sich aber nicht nur dem Armeedienst durch Desertion entzog, sondern gleichzeitig Republikflucht beging und damit sein gesamtes Lebensumfeld hinter sich ließ, ist davon auszugehen, daß er letztlich keine andere Möglichkeit sah, sich aus seiner $Z$ wangslage zu befreien. Bei seinem grundsätzlichen Bruch mit dem System, den die schwere Fahnenflucht zwangsläufig bedeutete, kalkulierte er bewußt mit ein, daß seine Handlung als politisches Delikt gewertet und daher im Falle des Scheiterns der Flucht um so schärfer sanktioniert werden würde ${ }^{96}$.

93 Bericht der Untersuchungskommission der 7. Panzerdivision, 14. Dezember 1960, BA-MA, VA-01/5966, o. Pag.

94 Allgemeine Erklärung der Menschenrechte vom 10. Dezember 1948, S. 41.

95 Vgl. S. $113 \mathrm{ff}$.

96 Die Qualifikation der schweren Fahnenflucht als Widerstand geht konform mit der rechtlichen Einschätzung gemäß Regelbeispiel in Paragraph 1 Absatz 1 Ziffer 1. e) des Strafrechtlichen Rehabilitierungsgesetzes (StrRehaG) vom 1. Juli 1997, wonach eine Verurteilung wegen eines ungesetzlichen Grenzübertritts der politischen Verfolgung diente. Gesetz über die Rehabilitierung und Entschädigung von Opfern rechtsstaatswidriger Strafverfolgungsmaßnahmen, S. 1614. 